\title{
INDUSTRY SHOULD COLLABORATE ON SCALE UP PROBLEMS
}

A lthough the laboratory end of biotechnology's stream of development enjoys the lion's share of attention, the scale-up stage of process development determines the feasibility of actually manufacturing a product. The sheer cost in time, equipment, and labor of scaling up presents a roadblock to the small specialist companies that yearn to become large manufacturing and marketing concerns. The ability to scale up, and its cost, will determine whether and how some of the finest new companies will survive through the manufacturing stage, let alone compete with the multinational giants in the world marketplace. The U.K.'s Department of Industry and the Science and Engineering Research Council should be applauded for their attempts to upgrade their country's capacity for industrial scale up through programs that support, respectively, the business and technological development of new companies (see BIO/TECHNOLOGY 1:834).

In the U.S., where specialty firm start-ups continue to blossom, there is only one integrated program to assist burgeoning contenders in scaling up individual projects: the Small Business Innovation Program (SBIR, see BIO/TECHNOLOGY 2:22). It may be unrealistic for small and medium-sized businesses to expect the U.S. government to provide assistance for scale up beyond the SBIR program, given the government's reluctance to form partnerships with private enterprise or form multilateral agreements between competing companies and itself. The National Science Foundation, which devotes about $\$ 4-5$ million to bioengineering through its Division of Chemical and Process Engineering, will commit its resources only to generic scale-up problems.

Specialist firms should explore consortium relationships that enable them to engage in joint $R \& D$ and benefit from the resulting technology and licenses while sharing the burden of research costs. The most likely place for joint development centers to form is near universities with strong bioengineering departments. But there should be no reason why companies cannot pool some of their own bioengineering talents with firms that might even compete directly in certain areas, as long as they settle confidentiality and proprietary matters in advance.

Those who scoff at the idea of collaborative scale up studies should look to the computer industry as a model. Fear of losing the international race for new computer technologies has motivated the formation of two important new collaborations between corporations. One collaboration, initiated by William C. Norris, chairman of Control Data Corp., with the cooperation of 18 top industry executives, is an organization that expects to conduct its own research with a $\$ 75$ million budget supporting 225 researchers by 1985 . Known initially as the Microelectronics \& Computer Cooperative (MCC), it is now under the presidency of retired Admiral Bobby R. Inman in Austin, Texas. Companies join MCC with a $\$ 200,000$ entry fee, then provide partial financial support for projects they choose to participate in for three or more years. In return, participants in each project receive licenses to the resulting technology for three years after the project's conclusion. At the end of those three years, the technology can be licensed by any company. Projects are administered by MCC and staffed by researchers lent by the companies, in addition to MCC staff scientists. The Justice Department may allow MCC to function without charges of anti-trust activity, because MCC is developing technology, not products for commerce. In addition, the government may not view MCC as restraining trade, because it does not contain three of the biggest manufacturers in the industry.

The computer industry has spawned a second cooperative effort, called the Semiconductor Research Corp. (SRC). It consists of 19 companies who support 40 research initiatives at 30 universities. Some members of SRC would like to expand into in-house $R \& D$ and develop a manufacturing facility.

Ambitious small and medium-sized firms should consider studying these and other models of cooperation to boost their production technologies. As some computer industrialists have painfully learned, it is much easier to stay ahead in high technology through long-range $\mathrm{R} \& \mathrm{D}$, than to strain to catch up with competitive Goliaths at home and abroad.

-Christopher G. Edwards

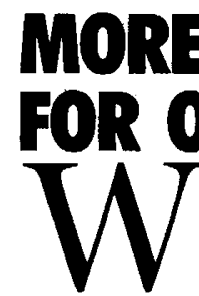
RESEARCH PAPERS OUR READERS

hen Macmillan Journals of London decided to launch a new publication called $\mathrm{BIO} /$ TECHNOLOGY, it considered several formats before deciding to combine original research data with news, features, and technology assessments. Instead of selecting an undistinguished journal format such as that seen most commonly in the learned society publications, it choose a format that combines tasteful design, four-color illustrations and photographs, and durable paper stock with a production method allowing extremely rapid publication of original research. The result is a journal that looks and functions more like Macmillan's proud progeny, Nature, and AAAS's Science than other academic journals.

In line with our perception of the expanding need for publishing distinguished biological research data with industrial implications, we are preparing to publish a larger number of shorter papers in future issues of BIO/TECHNOLOGY. Harvey Bialy, a molecular biologist who combines a highly imaginative research mind with a rare combination of editorial talents, has recently joined our staff as a research editor, to assist us in this task. We are also pleased to give credit to an editorial board which already has been instrumental in identifying areas of original research that the journal should cover. The scientific section of the editorial board includes:

(Continued on page 179) 